\title{
EN BUSCA DE SOLUCIÓN PARA EL SUFRIMIENTO: VIVENCIAS DE FAMILIARESEN EL PROCESO DE ENFRENTAMIENTO DE LA DEPRESIÓN
}

\author{
IN SEARCH OF TO THE SUFFERING: FAMILY M EM BERS EXPERIEN CES \\ DURING DEPRESION FACING PROCESS
}
MARÍA CONCEPCIÓN PEZO SILVA*, MAGUIDA COSTA STEFANELLI**, LUIZA AKIKO KOMURA HOGA***

\begin{abstract}
RESUMEN
Innumerables factores dificultan el diagnóstico y tratamiento precoz de la depresión. Esta investigación tuvo como objetivo describir cómo los portadores de depresión y sus familiares vivencian el proceso de búsqueda de ayuda para el sufrimiento provocado por esta enfermedad. El abordaje de la investigación fue la cualitativa y el método empleado la etnografía. Fue desarrollado el proceso de observación participante de 13 familias con un miembro afectado por la depresión. "En busca de solución para el sufrimiento" es el tema cultural que emergió de las narrativas y retrata el proceso de enfrentamiento de la depresión por la familia. La depresión causa sufrimiento cabien do a los profesionales de salud y a la propia sociedad la contribución para el diagnóstico precoz de la enfermedad y su debido tratamiento.
\end{abstract}

Palabras claves: Enfermedad mental, depresión, familia, asistencia a la salud, enfermería.

\begin{abstract}
Several factors make difficult to have an early depression diagnosis. This research had the purpose to describe how the persons affected by depression and their families, experience the process of searching for help while suffering by this illness. It is an ethnographic study developed through participant observation with 13 families affected by depression. The search of a solution related to suffering, is the central theme that emerged from the narratives and retracts the coping process of the families. The depression causes suffering and the professional is responsible for the early diagnosis and treatment.
\end{abstract}

Keywords: M ental health, depression, health care.

Recepción: 29.03.2004 Aceptación: 02.09.2004

\section{INTRODUCCIÓN}

La depresión ha sido el centro de atención de los profesionales e investigadores que actúan en el ámbito de la salud mental y también de los gestores de las políticas públicas de muchos países, pues se estima que afectaráa millares de personas en este siglo que se inicia. Tal atención es motivada por el hecho deafectar, no sólo a la persona enferma, sino que

\footnotetext{
* Enfermera. Profesora Principal de la Facultad de Enfermería de la Universidad Nacional "Pedro Ruíz Gallo"Lambayeque-Perú. Doctora en Enfermería grado obtenido en la Escuela de Enfermería de la Universidad de São Paulo (USP)-Brasil. E-mail: marypezo_2000@yahoo.com

** Enfermera. Profesora Titūlar de la USP. Orientadora del Programa de Doctorado de la Escuela de Enfermería de la USP. Asesora del Consejo Director del Instituto de Psiquiatría del Hospital de las Clínicas dela Facultad de M edicina de la USP-Brasil. E-mail: mcstefanelli@terra.com.br

*** Enfermera obstétrica. Profesora Asociada del Departamento de Enfermería M aterno-Infantil y Psiquiatrita de la Escuela de Enfermería de la USP - Brasil. E-mail: kikatuca@usp.br
} 
también a su familia, provocando serios problemas en la dinámica personal, familiar y social (Gentil Filho, 1999; O M S, 2001).

Personasquesufren dedepresión vivencian el inicio de la enfermedad de forma solitaria sin conseguir describir, claramente, el sufrimiento ocasionado por las primeras manifestaciones de comportamiento inherentes a la patología. Ellas consiguen expresar sus sentimientos de modo verbal o corporal aunque de forma vaga. Esto hace que los familiares y amigos de personas acometidas por la depresión tengan dificultades para identificar los primeros síntomas de esta enfermedad.

Las personas que sufren de depresión se caracterizan por la incapacidad de sentir placer con cosas que le proporcionaban tal sensación, permanecen aisladas, cabizbajas, silenciosas, síntomas que incomodan a las personas con las cuales convive. Su expresión facial es de tristeza, Ilora con facilidad y se presenta desarreglado en relación a su apariencia ehigiene personal.

La familia, frente a las observaciones realizadas, lo considera un "vagabundo" no sólo porque deja de dedicarse al trabajo sino también porque se aísla en su cuarto. Esta forma de interactuar con los deprimidos sólo cambia cuando es confirmado el diagnóstico mé dico a partir del cual sus síntomas pasan a ser vistos como propios de la enfermedad. No obstante en el curso que antecede al establecimiento del diagnóstico médico está presenteel sufrimiento de las personas que la padecen y de sus familiares (M oreno, Moreno, 1999; Laraia, 2000; Towsend, 2002).

La persistencia de los primeros síntomas físicos y emocionales del deprimido preocupa a los familiares. Éstos se sienten aprehensivos por desconocer la dimensión del problema y entran en un círculo vicioso caracterizado por muchos intentos de enfrentamiento, ensayos y errores, proceso que acompaña la búsqueda de solución para el sufrimiento causado por la enfermedad.

Se considera que el sufrimiento ocasionado por estaverdadera peregrinación por la que pasan las personas deprimidas y sus familiares podría ser aliviado y abreviado, si esteproceso fuese conocido por los profesionales de salud, sobre todo por aquellos que trabajan en el ámbito de la salud mental y de la clínica médica, la primera puerta de entrada de estos pacientes. Como forma de contribuir en el conocimiento de esta dimensión del problema, se llevó a cabo la presente investigación.

\section{OBJETIVO}

Describir cómo los portadores de depresión y sus familiares vivencian el proceso de búsqueda de ayuda para el sufrimiento provocado por esta enfermedad.

\section{MÉTODO}

Intentar comprender las prácticas cotidianas de un agrupamiento cultural por medio de una investigación, requiere la conciencia sobreel movimiento constante que existe en su interior. Laraia (2000) resalta quela visión de mundo está condicionada por la cultura, que hace el papel de una lente, por medio de la cual el hombre ve el mundo.

El desarrollo de la investigación etnográfica exige obligatoriamente la observación participante. Hammersley, Atkinson (1983) afirman que toda investigación social es una forma de observación participante. Ésta no esapenasuna técnica de investigación, mas sí un modo de ser en el mundo, humanístico einterpretativo, característico de sus seguidores.

En esta investigación se adoptó la etnoenfermería, constituidadecuatro fases (Leininger, 1991), siendo su principal característica la existencia dela fase dereflexión, incrementada a la tradicional observación participante realizada por los antropólogos.

La primera fase, la de observación primaria y escucha activa - o sin participación activa, ocurrió al observar familias que interactuaban en cuanto aguardaban su atención 
médica. O casiones en que conversaban sobre vivencias comunes en la convivencia con la persona deprimida - dificultades en la marcación de consultas, acceso a la institución, entre otros.

Las fases subsiguientes, observación primaria con alguna participación, y la departicipación con alguna observación, se siguieron en el mismo escenario. Fue posibleconocer personas con depresión y sus familias y obtener al gún grado defamiliaridad con ellos, marcándose visitas domiciliarias, para la observación del contexto familiar y realización de las entrevistas. Se siguieron las premisas básicas de la investigación etnográfica, en relación a la actitud de asombro de lo que era observado.

Da M atta (1993), M orse (1998) alertan que el presupuesto de la familiaridad exige conocimiento y proximidad, sin embargo éstos deben ser superados cuando se utiliza la óptica de la antropología social. Para mantener el carácter científico se observó este presupuesto. La familiaridad generó ben eficios a la investigación, pues permitió profundizar en las cuestiones que afectan la vida de familia.

Durante la observación participante se mantuvo el diario de campo para la anotación de las peculiaridades observadas, al mismo tiempo en que se fotografiaba escenarios considerados importantes para elucidar y ejemplificar los datos de esta investigación.

En la cuestión de la interpretación, la fase final, la de reflexión de la etnoenfermería, permitió la reconfirmación de los resultados con las familias, lo quehizo posiblela atribución de significados a los datos, dentro de su propio contexto (Leininger, 1991).

\section{Escenario estudiado}

El escenario estudiado fue el ambiente familiar de los enfermos portadores de depresión con tratamiento. Éstosfueron identificados en Ios consultorios externos del Instituto dePsiquiatría del Hospital de las Clínicas de la Fa- cultad de M edicina de la Universidad de São Paulo (I pq HCFM USP). En las residencias, donde en algunos casos había además del paciente otro miembro con depresión, se llevaron a cabo los procesos de observación participante y las entrevistas. El desarrollo de la observación participante fue esencial para comprender, in locus, la dinámica interaccional entre los miembros de la familia. La obsevación ocurrió también en la sala de espera de los consultorios.

El Instituto dePsiquiatría es una delas instituciones que componen el complejo Hospital de las Clínicas. Se trata de un hospital docente que posee diferentes ambientes de atención tales como consultorios externos, internamiento, hospital de día, psicoterapia, servicio de atención domiciliaria, centro de rehabilitación y hospital de día, entre otros.

En los consultorios externos se desarrollan acciones administrativas, atención individual, consulta de enfermería, atención de emergencia, sesiones grupales de orientación para el uso de los medicamentosindicados, charlas y exposición de videos en la sala de espera sobreasuntos directamenterelacionados a trastornos mentales y cuidados con la salud en general. La atención es eminentemente ambulatoria, ya que las internaciones están restringidas a los portadores detrastornos mentales que requieran de tal medida.

Los pacientes residían en zonas periféricas de la ciudad y eran miembros de familias de medio y bajo ingreso. Éstas presentaban seme janzas relativas a las condiciones dela morada, transporte, socioeconómicas, entre otras. Las fases del proceso de observación participante se efectuaron en el transcurso de las visitas realizadas a las residencias. Éstas eran en su mayoría simples, al gunosmoraban en peque ños departamentoslocalizados en el centro de la ciudad, no obstante, en condiciones muy precarias.

Fueen losmomentos demayor relajación, en las pausas realizadas en las entrevistas para tomar los "cafecitos" que habitualmente eran servidos, quese pudo conocer aspectos gene- 
rales delasfamilias estudiadas, sobretodo sus principales dificultades y problemas enfrentados en el proceso de búsqueda de atención para el enfermo con depresión. La dinámica familiar, los diferentes papeles desempeñados por sus miembros, las alianzas existentes, el tipo de comunicación predominante y el desarrollo de los quehaceres domésticos fueron algunos de los aspectos observados. Estos momentos propiciaron el desarrollo de la relación terapéutica con las familias, por medio del ofrecimiento deayuda, disponibilidad para escuchar sus demandas y aclarar sus dudas.

\section{Informantes y entrevistas}

En esta investigación los nativos de la cultura estudiada fueron los miembros de las familias que tenían uno o más de sus miembros afectados por la depresión. Se consideró que estas familias constituían un agrupamiento cultural por el hecho de tener en común el soporte institucional, en este caso el IPq, así como las creencias, valores, prácticas relativas a la enfermedad y la experiencia familiar vivida en relación a la convivencia con una persona que sufre de depresión.

Los informantes de esta investigación fueron los componentes de 13 familias de ambos sexos, cuyas edades oscilaban entre los 18 y 50 años, con los cuales se realizaron las entrevistas etnográficas. Ellos eran integrantes defamilias quetenían uno o más desusmiembros con diagnóstico médico de depresión unipolar en tratamiento ambulatorio en el IPq. Al inicio, al preguntarle a los miembros de las familias si deseaban ser entrevistados individualmente $o$ en conjunto, todos optaron por la segunda alternativa.

Las familias estudiadas eran del tipo nuclear (siete), extensas (dos), con madre ehijo (dos) y esposos sin hijos (dos). Por el hecho de haberse efectuado las entrevistas en las residencias, en algunas de ellas hubo la participación de otras personas. El deseo de inclu- sión de sus relatos fue respetado, pues eran datos que enriquecían los contenidos de las entrevistas.

Seoptó por el tipo deentrevista no estructurada según Fontana; Frey (1994). Para éstos la meta principal de este tipo de entrevista es la comprensión sobre al go que se quiere conocer. Debemos considerar que cada persona poseesu propia historia social y cada cual asumeuna perspectiva individual en el mundo. Los investigadores deben ser suficientemente sensibles a las diferencias y peculiaridades individuales respetándolas y efectuando las preguntas "persona a persona", para obtener una respuesta amplia y completa.

Basadas en estas premisas, se realizaron entrevistas con explicación previa sobre lo que se estaba deseando comprender. La esencia de las preguntasfue orientada por el objetivo propuesto en este estudio, o sea, cómo los portadores de depresión y sus familiares vivencian el proceso de búsqueda de ayuda para el sufrimiento provocado por esta enfermedad.

Todas las entrevistas fueron realizadas, grabadas y transcritas íntegramente por una de las investigadoras. Los datos obtenidos durantela observación participantefueron registrados en el diario de campo. En él se anotaron datos relativos al transporte utilizado, el metro y el ómnibus, la constitución familiar, las condiciones de habitación, fuente de ingresos, hábitos de salud, recreación y acceso a las instituciones. La recolección de datosfuerealizada en el 2002 y el promedio de duración fue de dos horas y treinta minutos por familia.

\section{Análisis de los datos}

El proceso de análisis de los datos fue realizado en el transcurso del desarrollo de las entrevistas. Las lecturas repetidas de las transcripciones permitieron la identificación delos aspectos relacionados con la experiencia cotidiana de las familias estudiadas, lo que permitió la construcción de los temas culturales. 
Temas culturales son enunciados con alto grado de generalidad (Leininger, 1991). Ellos pueden revelar la esencia de lo que ocurre en la cultura, en estecaso, la forma cómo es vivido el proceso de búsqueda de ayuda para el sufrimiento provocado por esta enfermedad.

El rigor científico de la investigación fue respetado según Leininger (1991). Durante todo el proceso de investigación se mantuvo la necesaria apertura al aprendizaje sobrealgo desconocido, por medio de la actitud de "escucha activa y aprendizaje genuino" en relación a aquello que era observado y narrado. Por medio de esta actitud fue posible aprender sobre los significados, las expresiones, creencias y valores, formas de conducta, tratando de evitar la actitud etnocéntrica del investigador. Ésta fue evitada durante el proceso de aprendizaje relativo a las conductas tomadas por los familiares en la búsqueda de solución para el sufrimiento ocasionado por la depresión.

Seprestó atención constantea la comprensión de la razón de las conductas de los familiares, así como a la fidelidad de los datos. Por tal motivo las entrevistas grabadas fueron escuchadas varias veces y transcritas íntegramente. La descripción cultural estuvo basada en la perspectiva deaquel queconvivecon un familiar deprimido en su cotidiano, pues éste es un tipo de conocimiento útil para los profesionales que necesitan comprender las vivencias de las personas para proponer una asistencia culturalmente congruente.

Los aspectos éticos fueron seguidos de acuerdo a lo preconizado en la Resolución 196/96 (Brasil, 1996). Se tomó especial cuidado en preservar el sigilo de los datos así como el anonimato y bienestar de las personas participantes del estudio. La investigación sólo fue realizada después de la obtención de la aprobación de la Comisión de Ética de la institución involucrada, dela firma delosinformantes respecto al consentimiento informado y de la autorización para grabar las entrevistas.

\section{PRESENTACIÓN DE LOS RESULTADOS}

El tema cultural "En busca de solución para el sufrimiento" constituye el primer paso seguido por el paciente y sus familiares cuando comienza a enfrentar el sufrimiento ocasionado por los signos y síntomas de la depresión aún no reconocida como enfermedad.

Los informantes de este estudio fueron identificados según el grado de parentesco en relación al paciente $(P)$ y a cada familia se le atribuyó un número (F1, F2...), conforme el ejemplo a seguir:

“Uno imagina fatiga, cansancio, eh... Ud. piensa indisposición..., pero no en una enfermedad (Esposo F1). Las personasno entienden... piensan que uno es perezosa. (Madre-P F7).

Los familiares comienzan a recibir sugerencias para la procura de recursos al ternativos disponibles en la comunidad, como los curanderos y centros religiosos. Éstos son recomendados como fuentes de alivio delossíntomas somáticos y psíquicosque afectan a los que padecen de depresión y buscados preliminarmente al tratamiento médico.

"Ud. procura iglesia, centro de macumba, en fin todo lo queayudea al iviar... en cuanto no tenga la certeza de quese trata de una depresión..." (M adre-P F1).

El nivel de ansiedad de los familiares aumenta a medida que el estado de salud del enfermo se agrava. Esto provoca mucha tensión en el ambiente familiar e impulsa a la búsqueda de recursos de alivio disponibles, hasta de aquellos evaluados como contrarios a las creencias religiosas. Aún no acreditando en la eficacia de esos recursos, las familias los utilizan en razón de las insistentes recomendaciones realizadas por parientes y amigos.

“... Procuré de todo en aquella época, hasta candomblé. Algunos de esos trabajos me ayudaron. Eso que eran completamente 
contrarios a mi formación religiosa... mas decidí flexibilizarmey aceptécualquier tipo de ayuda" (Hijo-P F12).

El proceso de búsqueda de ayuda en las instituciones de salud se inicia por voluntad propia o cuando son estimulados por otros familiares. El primero a ser procurado es el clínico general, quien mayormente los encamina hacia otros especialistas $y$, por fin, al psiquiatra.

La búsqueda de asistencia médica generalmente es motivada por la presencia de síntomasfísicos como la hipertensión, la sensación de sofocación, disnea, dolores precordiales, taquicardias. Los primeros recursos de asistencia médica que procuran son los servicios de emergencia, clínicas o puestos de salud. Recurrir a uno u otro recurso depende de las condiciones deaccesibilidad a ellas, sobretodo de los recursos financieros disponibles en aquel momento de urgencia.

“... en un día de madrugada... pasémal, me dio aquella cosa horribleen el pecho... Hablécon mi marido que si es para procurar el hospital, hoy era el dia. Fue así queacudí al puesto de salud" (M adre-P F1).

La búsqueda de ayuda médica evidencia la percepción dela familia a cerca dela ocurrencia de síntomasque precisan ser aclarados. El enfermo y su familia acuden a las diversas especialidades con el fin de encontrar alguna causa orgánica a los síntomas que presentan. Estos no llegan a manifestar su descontento de forma clara cuando los médicos no encuentran soluciones para su problema. No obstante juzgan que esta búsqueda inicial es necesaria para el alivio de los síntomas orgánicos referidos por la persona enferma.

“N osotros ya fuimos al psiquiatra... mas vea! ya pasamos por tantos médicos... clínico general, psiquiatra, psicólogo, ginecólogo... en fin... mi mujer es el tipo de persona que se queja de todo" (Esposo-P F7).
El diagnóstico de la depresión es confirmado, a veces, de forma eventual. Fue el caso de una familia que, al buscar asistencia psiquiátrica para el tratamiento de otro familiar, descubrió que la madreera portadora de de presión.

“... Yo no percibí queella estaba con depresión... fuecon el tratamiento de mi hijo que mi esposa conversó con la médica a cerca de su estado, ahí fue diagnosticada su de presión" (Esposo F6).

Los prejuicios relativos a la enfermedad psiquiátrica dificultan el enfrentamiento de este problema. El portador de depresión y su familia no asumen los síntomas presentados como señal de enfermedad mental, situación que origina postergación del tratamiento.

"Ella se rehusaba a ir al psiquiatra porque no quería admitir su locura... decía queno era una débil mental... Eso era un problema, porque ella se resistía a procurar ayuda" (Esposo F4).

La persona afectada por la depresión se percibe diferente y se siente discriminada no sólo por los médicos, familiares y amigos, sino por la sociedad en general.

“... hablar de depresión da estremecimiento, la impresión dequeeres un loco menor, tú no quieres que las personas te miren, porque te tratan con diferencias... sea el médico, o las personas... Como aquello queda grabado en el inconsciente, las personas se castigan por el hecho de estar en psiquiatría" (Esposa-P F2).

El establecimiento definitivo del diagnóstico de depresión determina el inicio de su tratamiento. La falta de conocimiento de los recursos disponibles para el tratamiento de la enfermedad, que no se restringe a los medicamentos, produce incomodidad en la familia, sobre todo cuando el enfermo es solicitado a asistir a las consultas posteriores con 
cierta frecuencia. Esta laguna dificulta la aceptación inicial del tratamiento médico psiquiátrico, constituido básicamente por psicoterapia y medicamentos. Muchas veces los familiares demuestran dificultad para aceptar el envolvimiento que se establece entre profesionales y clientes durante el tratamiento psicoterapéutico.

“... con el médico yo tenía la oportunidad de hablar mis conflictos... sin embargo surgieron celos de toda mi familia, inclusive de mi marido... tenían la sensación de que estaba traicionando a mi marido con el doctor, que en aquella época estaba separado de su esposa... aquello me quitó la libertad para decirle todo lo que sentía..." (MadreP F1).

Muchosfamiliares desconocen el hecho de que la persona con depresión tiene que ser tratada con medicamentos. Consecuentemente, el seguimiento preciso de las indicaciones médicas se torna difícil en razón de los prejuicios. Existe el temor del surgimiento de dependencia y de los efectos colaterales provocados por el uso de los medicamentos psiquiátricos, desconocimiento que, al no ser subsanado por medio de las orientaciones apropiadas, puedellevar al abandono del tratamiento.

“... tuveinsomnio por añossin embargo me resistía a tomar algo... pensaba que el remedio es dañino porque causa dependencia... como puede ver uno tiene prejuicios en relación al remedio" (Esposo F2).

A pesar de estos contratiempos, muchos enfermos reconocen la efectividad de los medicamentos en la remisión de sus síntomas. Algunos llegan hasta el estado de dependencia porque, en general, usan el medicamento como elemento de apoyo o seguridad para el enfrentamiento de las diversidades vivenciadas. Para muchos enfermos se torna casi imposible el enfrentamiento de situaciones ad- versas en ausencia del medicamento utilizado como soporte.

“... cuando tomé aquel medicamento, pareceque tuvo quever con mi ego. Medaba la sensación de estar despertando de una pesadilla..." (Esposa-P F2).

"Del tranquilizanteno tenía másnecesidad, sin embargo lo tengo en la bolsa para tomarlo cuando pasealgunacontrariedad. En vez dedejar que aquello meafecte, yo tomo la mitad y paso bien el dia..." (M adre-P F4).

La convivencia prolongada con el tratamiento psiquiátrico proporciona, a la persona enferma, habilidad para visualizar aspectosque, a su parecer, contribuyen en la mejoría de su cuadro. Fueron mencionados, entre otros, la eficacia de la psicoterapia de grupo asociada al tratamiento farmacológico, evaluadas como importantes porque ayudan a disminuir la demanda de pacientes en los hospitales psiquiátricos.

La terapia grupal, según los propios enfermos, permitiría la convivencia mutua, y el poder compartir experiencias y síntomas semejantes. Consideran que ella proporciona vivencias favorables para el tratamiento, si ocurren de forma organizada y si es dirigida por el médico psiquiatra u otro profesional especializado de la institución.

“... si se asociara la terapia al tratamiento psiquiátrico la demanda de pacientes sería menor, porque ella da fuerzas para luchar, para tener percepción de uno mismo. No sési eso es una utopía, pero de ser posible, sería lo ideal" (Esposa-P F2).

“... es una oportunidad de hablar con gente queestá hablando la mismalengua, no existe otra oportunidad, ¿entendió?

Las informaciones disponibles en los medios de comunicación proporcionan algún grado de conocimiento a los enfermos y sus familiares. Son conocimientos muy valoriza- 
dos, pues consideran que el cuestionamiento dirigido a los médicos es difícil en ausencia de un conocimiento previo. Por medio de ellos, las personas enfermas y sus familiares no sólo aclaran sus dudas sobre los remedios y la enfermedad sino que cuestionan lo que identifican como errores. Así, pasan a representar el papel de agentes activos en ese proceso.

“... es difícil que un paciente cuestione las cosas con el médico iporqueellos no aceptan!Yo llegué y argumenté... ya conozco el proceso dela depresión... Yo habléeso porque conocía... si yo no conociese, ¿yo iba a hablar? Apenas me iba a conformar con lo que me dijesen" (Esposa-P F2).

La condición de pobreza y carencia económica ocasiona la vivencia de experiencias traumatizantes en la fijación del día de la consulta, el mismo que implica el enfrentamiento de largas filas formadas desde las primeras horas de la madrugada, sin la garantía de la obtención de un cupo. Una vez superado este obstáculo continúa el proceso de espera, en los consultorios externos, el día para el que fue citado.

Súmanse a las vivencias, las dificultades provocadas por la escasez de médicos y demás profesionales, cuyo conjunto resulta en la precariedad de la atención psiquiátrica en los servicios públicos de salud.

“... La atención es precaria, principalmente en los alrededores donde vivo, eso me deja desanimado y depresivo..." (Esposo-P F7).

Las familias perciben que consiguen poca mejoría con las atenciones prestadas en los servicios de emergencia o puestos de salud. Consideran que esto pueda deberse a la deficiencia en la asistencia ofrecida en los hospitales públicos queen general es de modo masificado. La atención recibida en el Instituto de Psiquiatria del HCFM USP, a pesar de ser público y atender a un número elevado de pacientes, les permitió establecer la diferencia de los resultados obtenidos en esa Institución.

“Pasé por un clínico general, después, por un psiquiatra, sin embargo no fue un tratamiento tan bueno cuanto el querecibí en el Hospital de las Clínicas (Instituto), porquefui a un puesto de salud... Por más que conozcan el asunto, no es una cosa quedan secuencia... el volumen de personas es muy grande" (Esposo-P F13).

\section{DISCUSIÓN Y CONSIDERACIONES FINALES}

Los resultados de esta investigación demuestran que el enfrentamiento inicial dela depre sión por la familia posee al gunas características, entreellas la dificultad para reconocer que se trata de una enfermedad mental y la búsqueda de tratamiento en instituciones de salud y recursos alternativos de la comunidad. Es una trayectoria caracterizada por el tema cultural "búsqueda de solución para el sufrimiento", que constituyeuna de las vivencias de las familias que conviven con la depresión.

La dificultad para el reconocimiento de la depresión como problema de salud mental ocurre también en otros contextos socioculturales, como consta en el estudio realizado en una comunidad peruana por Perales, M ontoya, Sogi (1995). Estos investigadores comprobaron que la depresión fue reconocida como problema desalud mental apenas por el 1,6\% de sus entrevistados. Las personas deprimidas acostumbran a no quejarse de los trastornos físicos propios de la enfermedad, lo que hace que la búsqueda de solución al sufrimiento causado por la depresión se dé a partir de las quejas somáticas expresadas por los enfermos (Travelbee, 1982) y que ésta sea, en primer lugar, en los médicos clínicos generales, sobre todo aquellos que laboran en los puestos de salud, hospitales o clínicas particulares. 
El contenido relativo a las enfermedades mentales debeser obligatorio en los cursos de las profesiones del área de la salud, inclusive de la enfermería (Stefanelli et al., 1996), sobre todo el de la depresión. Está proyectado por investigadores que un mayor número de personasanivel mundial sufrirádedepresión a través del presentesiglo que se inicia (Gentil Filho, 1999; OM S; M cCabe, 2002).

Los profesionales, a su vez, los orientan a diversas especialidades de la clínica general, por la dificultad para identificar síntomas psiquiátricos, inclusivelos derivados dela depresión (Stefanelli et al., 1998). A esto se atribuye el retraso para el inicio del tratamiento en un gran contingentedeenfermos, quelos perjudica. La prevalencia de personas atendidas en el ámbito dela atención primaria en salud que presentan síntomas de depresión varía entre 18 a $83 \%$, en su mayoría consecuentes de alguna enfermedad orgánica (Fraguas; Figueiró, 2001).

Cuando finalmentelosenfermos y respectivos familiares son orientados al psiquiatra se resisten a la idea de procurarlos en razón de los prejuicios que poseen en cuanto a la enfermedad mental. En general, la persona que sufre de depresión busca ayuda psiquiátrica de manera individual, pues teme la incomprensión familiar y no desea ser fuente de preocupación para su familia, la misma que, según ellos, tiene dificultad para aceptar esta enfermedad. El desconocimiento y prejuicio relativos a la enfermedad mental constituyen las princi pales barreras de la participación familiar en el tratamiento deenfermos portadores de trastornos psiquiátricos (Yacubian; Neto, 2001). Deestemodo, la búsqueda del profesional específico se retarda y consecuenteel tratamiento se posterga, aspectos que se asocian a la vivencia de la depresión de modo solitario.

Constituyen también blanco delos prejuicios, la resistencia a iniciar y dar continuidad al tratamiento farmacológico. Ello se debe al temor de una posible dependencia, al surgimiento de efectos colaterales así como a las burlas de las que son víctimas. En la mayoría de los casos el tratamiento farmacológico es interrumpido cuando los enfermos que sufren de depresión son llamados drogados, por sus familiares, debido a los efectos colaterales que le produce la ingestión de los antidepresivos.

El enfrentamiento de experiencias traumatizantes por parte de las personas con depre sión y susfamiliares indicala necesidad de ofrecimiento de informaciones adecuadas sobrela enfermedad. $\mathrm{H}$ ay evidencias que los periodistas y los diversos medios decomunicación de sarrollan importantepapel en la diseminación de informaciones relativas a la enfermedad (Fundación Americana para la Prevención del Suicidio; Comité de radio, 2003).

La enfermería tiene que actuar en pro de mayor adherencia de los pacientes deprimidos al tratamiento y a los programas psicoeducativos. Se les debe proporcionar un tipo de orientación clara, amplia y decodificada en relación a la importancia del seguimiento del tratamiento prescrito y cuidados para evitar los perjuicios consecuentes de la enfermedad (Andrade, 1999; Stefanelli, 2003) y contribuir para desmitificar y disminuir lostabúes y prejuicios existentesfrentea la enfermedad mental, inclusive la depresión.

Esta investigación permitió constatar que las familias que poseen uno o más de sus miembros afectados por la depresión no poseen condiciones para procurar tratamiento de modo adecuado. Este dato indica la necesidad deaclarar amplia y adecuadamente a la población en relación a los recursos disponibles para el tratamiento psiquiátrico. En ese trabajo se han de incluir aspectos relativos a los síntomas característicos de la depresión, dado quelasfamilias estudiadas tuvieron dificultades para identificarlas. Estehecho provocó la realización de un verdadero "vía crucis" entreel surgimiento inicial de los síntomas de depresión y el inicio de su tratamiento, con todas las consecuencias del proceso sufridas por los enfermos víctimas de la depresión y sus familiares. 
Evitar que las familias sufran las consecuencias de la falta de atención es responsabilidad de los profesionales involucrados, sobre todo a la enfermera, que hace la intermediación de los pacientes con el tratamiento médico, cabe hacer la contextualización sociocultural del cuidado. Sólo así será posible prestar un cuidado sociocultural congruente (Leininger, 1991). Por tanto, las enfermeras tienen que mantener la alianza entre el cuidado de enfermería y el bagaje cultural delas personas y sus familias (Mulhall, 1996).

La enfermería tiene un papel primordial en el proceso de orientación de esos familiares. La asistencia a la familia tieneque ser efectuada de manera personalizada, considerando sus características relativas a la inserción social, bagajecultural, entreotrosaspectosque influencian las vivencias del proceso saludenfermedad. Esa forma de cuidar es esencial para que las reales demandas por cuidados sean atendidas (Chen, 1998).

En este artículo se presentaron resultados de investigación desarrollada en determinado contexto sociocultural. Se cree, entre tanto, que las vivencias iniciales de familiares relativas a la convivencia con una persona deprimida puedan ser comunes a otros agrupamientos culturales. Se vivencia actualmente el tiempo postmoderno (Taschner, 1999), en que hay una amplia y generalizada difusión de creencias, valores y prácticas sobre determinado tema. Es la tecnología que permite el compartir, en tiempo real, ideas que son mutuamente influenciables.

\section{REFERENCIAS BIBLIOGRÁFICAS}

AndradeA.C.E. (1999). A abordagem psicoeducacional no tratamento do transtorno afetivo. Rev. Psiq. Clin. 26(6):12-16.

Atkinson, P.; H ammersley, M . (1998). Ethnography and participant observation. In: Denzin, N .K.; Lincoln, Y.S. Strategies of qualitative inquiry (pp. 110-36). Thousand O aks: Sage.

Brasil (1996). Conselho Nacional de Saúde. Resolução n.196 de 10 de outubro de 1996. Diretrizes e nor- mas regulamentadoras de pesquisa em seres humanos. M inistério da Saúde. Mundo saúde, 21: 52-61.

Chen, M. Y. (1999). The effectiveness of health promotion counseling to family caregivers. Public Health Nurs. 16(2),125-32.

Da Matta, R. (1993). Trabal ho decampo. In: Relativizando: uma introdução a antropologia social (pp. 143-73). Rio de Janeiro: Rocco.

Fontana, A., \& Frey, J.H . (1994). Interviewing: the art of science. In: Denzin, N .K., \& Lincoln, Y.S. H andbook of qualitative reserch (pp. 361-76). Thousand Oaks: Sage.

FráguasJR R., \& Figueiró J.A.B. (2001). Depressões em medicina interna e em outras condições médicas: depressões secundárias. In: Depressões secundárias: peculiaridades da depressão no contexto médico não-psiquiátrico. (pp. 3-10). São Paulo: Atheneu.

Fundación Americana para la Prevención del Suicidio. Asociación Americana de suicidiología. Centro de Políticas Públicas de Annenberg. Informando un suicidio: recomendaciones para los medios de comunicación. [online]. Traducido por el Comitêde Radio. Disponible en <http://www.comitederadio. com.pe> (14 de out. 2003).

Gentil Filho, V. U ma leitura anotada do projeto brasileiro de reforma psiquiátrica. Revista USP, v. 43, p. 6-23, 1999.

Hammersley, M. \& Atkinson, P. (1983). Ethnography: principles in practice. London: Tavisttock.

Laraia, M.T. \& Stuart, G.W. (2000). Enfermagem psiquiátrica: principios eprática. Porto Alegre: Artmed.

Leininger M .M . (1991). Culturecare diversity and universality: a theory of nursing. New York: National League for Nursing Press.

M ccabe S. (2002). The nature of psychiatric nursing: theintersection of paradigm, evolution and history. Arch Psychiatr Nurs 2002; 16(2):51-60.

M oreno, R.A. \& M oreno, D.H . (1999). Prática psiquiátrica em consultório. Rev. USP. 43(1): 44-53.

Morse, J.M . (1998). Designing funded qualitative research. In: Denzin, N.K. \& Lincoln, Y.S. Strategies of qualitativeinquiry (pp. 56-85). Thousand Oaks: Sage.

Mulhall, A. (1996). Anthropology, nursing and midwifery: a natural alliance? Int. . Nurs. Stud. 33(6), 629-37.

Organização Pan-Americana Da Saúde. Organização Mundial Da Saúde (2001). Relatório sobre a saúde no mundo: 2001 - saúde mental, nova concepção, nova esperança. Genebra: Organização Panamericana de Saúde/Organização M undial de Saúde.

Perales, A., M ontoya, A., \& Sogi, C. (1995). Linderos sociales y culturales para la salud mental comuni- 
tária. Lima: Instituto Nacional de Salud Mental "Hideyo Noguchi y Universidad Particular Caytano Heredia.

Stefanelli M.C., Bardelli M .H., Silva, I.A. (1998). Percepções e reações do pessoal da equipe de enfermagem, de hospital geral, às manifestações de distúrbio emocional depacientesinternados. Cogitare Enferm; 3(1):97-104.

Stefanelli M.C., Rolim M .A., Teixeira M .B., Barros S., Fukuda I.M .K., Forcella H .T., et al. (1996). Integração dos conceitos de saúde mental nos cursos de graduação em Enfermagem Rev Paul Enferm; 15 (1/3):51-65.
Stefanelli M.C. (2003). O profissional e a família em situação dedoença. Ci Cuidado Saúde; 2 (supl):502.

Taschner, G. B. (1999). A pós-modernidade e a sociologia. Revista U SP. 42(1), 6-19.

Townsend, M. C. (2002). Enfermagem Psiquiátrica: conceitos de cuidados. Rio de Janeiro: Guanabara.

Travelbee, J. (1982). La comunicación con los pacientes. In: _._. Intervención en enfermería psiquiátrica (pp. 47-94). Bogotá: Carvajal.

Yacubian, J. \& Lotufo N eto, F. (2001). Psicoeducação familiar. Fam Saúde Desenvol. 3(2), 98-108. 